\title{
Cloud advection model of solar irradiance smoothing by spatial aggregation $\odot$ (c)
}

Cite as: J. Renewable Sustainable Energy 13, 033704 (2021); https://doi.org/10.1063/5.0050428 Submitted: 15 March 2021 • Accepted: 26 May 2021 • Published Online: 23 June 2021

(iD) Joseph Ranalli and (D) Esther E. M. Peerlings

COLLECTIONS

F This paper was selected as Featured

SCI This paper was selected as Scilight
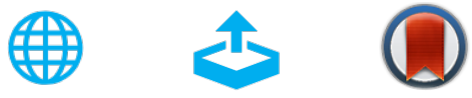

\section{ARTICLES YOU MAY BE INTERESTED IN}

Solar forecasting model focuses on shorter time scales to address irradiance variability Scilight 2021, 261103 (2021); https://doi.org/10.1063/10.0005503

A solar resource classification algorithm for global horizontal irradiance time series based on frequency domain analysis

Journal of Renewable and Sustainable Energy 13, 036102 (2021); https:// doi.org/10.1063/5.0045032

Adaptive fractional order control of doubly fed induction generator based wind energy conversion system under uncertainty

Journal of Renewable and Sustainable Energy 13, 033311 (2021); https://

doi.org/10.1063/5.0041047

\section{Scilight}

Summaries of the latest breakthroughs in the physical sciences 


\title{
Cloud advection model of solar irradiance smoothing by spatial aggregation $\odot$ ()
}

\author{
Cite as: J. Renewable Sustainable Energy 13, 033704 (2021); doi: 10.1063/5.0050428 \\ Submitted: 15 March 2021 • Accepted: 26 May 2021 • \\ Published Online: 23 June 2021

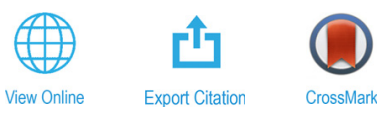

Joseph Ranalli, ${ }^{1, a)}$ (D) and Esther E. M. Peerlings ${ }^{2}$ (D)

\author{
AFFILIATIONS \\ ${ }^{7}$ Penn State Hazleton, Hazleton, Pennsylvania 18202, USA \\ ${ }^{2}$ German Aerospace Center (DLR), Institute of Networked Energy Systems, Carl-von-Ossietzky-Str., 15, 26129 Oldenburg, Germany
}

${ }^{a)}$ Author to whom correspondence should be addressed: jar339@psu.edu

\begin{abstract}
Solar generation facilities are inherently spatially distributed and therefore aggregate solar irradiance in both space and time, smoothing its variability. To represent the spatiotemporal aggregation process, most existing studies focus on the reduced correlation in solar irradiance throughout a plant's spatial distribution. In this paper, we derived a cloud advection model that is instead based upon lagging correlations between upwind/downwind portions of a distributed plant, induced by advection of a fixed cloud pattern over the plant. We use the model to calculate a plant transfer function that can be used to predict the smoothing of the time series. The model was validated using the distributed HOPE-Melpitz measurement dataset, which consisted of 50 solar irradiance sensors at $1 \mathrm{~s}$ temporal resolution over a $3 \times 2 \mathrm{~km}^{2}$ bounding area. The initial validation showed that the advection-based model outperforms other models at predicting the smoothed irradiance time series during manually identified, advection dominated conditions. We also conducted validation on the model against additional advection dominated periods in the dataset that were identified algorithmically. The cloud advection model's performance compared well to models in literature, but degraded slightly as larger cross-wind plant distributions were investigated. The results in this paper highlight the need to incorporate advection effects on spatial aggregation during advection dominated conditions. Future development of spatiotemporal aggregation models is needed to unify advective models with existing correlation reduction models and to identify regimes where each dominate.
\end{abstract}

Published under an exclusive license by AIP Publishing. https://doi.org/10.1063/5.0050428

\section{INTRODUCTION}

Electricity generation by solar photovoltaics (PV) remains an important part of a renewable energy based future for the world and plays a key role in many countries' roadmaps to mitigating climate change. PV relies on the incoming solar irradiance as an energy resource, so PV generation reflects any variability in the solar irradiance. Since PV generation is spatially distributed within an environment, forecasts of solar irradiance must be able to identify both the spatial and temporal nature of this variability. While variability due to the daily solar cycle is deterministic and can be accounted for, cloudand weather-induced variability is more difficult to describe and forecast. ${ }^{1}$ Nonetheless, forecasts are a critical element of grid management and are necessary to enable electrical system operators to prepare for ramping events. Thus, it is important to develop better understanding of solar variability as the quantity of grid-connected solar generation continues to increase. ${ }^{2}$

Although generation facilities are by nature spatially distributed, a site's solar irradiance monitoring and reference measurements are typically made by only a small number of point sensors. It is well established in the literature that the variability in solar irradiance is spatiotemporally smoothed by distributed collection facilities. ${ }^{3}$ It would be cost prohibitive to increase the number of sensors to monitor the spatiotemporal variability, so there is a need to develop models that describe how variability of a small number of point solar irradiance measurements leads to the plant's smoothed output variability. PV generation takes many forms, from relatively localized utility scale plants with rated capacities up to hundreds of megawatts, down to distributed rooftop PV installations as small as a few kilowatts. Therefore, it is desirable for models of aggregate variability to be flexible and able to accommodate a great variety of plant configurations.

\section{BACKGROUND}

There is a great deal of literature dealing with the spatiotemporal variability in solar irradiance, as well as the effects of spatial aggregation on variability in particular. For a starting point on the topic, readers may consider detailed reviews of variability and variability 
reduction due to both spatial and temporal aggregation, which are available from Perez et al. ${ }^{4}$ and Lohmann. ${ }^{1}$

A common way to characterize variability is via the clear-sky index, $k_{c}$. Clear-sky index is defined as the ratio between actual global horizontal irradiance (GHI) and GHI modeled for a clear-sky condition. The clear-sky index eliminates the effects of the diurnal cycle from the time series. It is typical to calculate increments (also termed "ramp rates") in the clear-sky index time series, $\Delta k_{c}$. These ramp rates are computed as the difference in clear-sky index over a certain time interval, $\Delta t$ (also termed "time span" or "time lag" in literature). This is represented as the quantity, $\Delta k_{c_{\Delta t}}{ }^{4,5}$ Note that it is important to distinguish between the time interval used to calculate the ramp rate, $\Delta t$, and temporal averaging of the time series, which can also represented by a certain time period, $\tau$. The standard deviation, $\sigma$, of the ramp rate time series is then used as a metric to represent the magnitude of variability as in Perez et al,

$$
\text { Variability }=\sigma\left(\Delta k_{c_{\Delta t}}\right) .
$$

An additional quantity, termed the relative output variability $(\mathrm{ROV}),{ }^{3}$ may be used to characterize the degree to which variability is reduced by spatial or temporal aggregation. ROV is measured relative to some reference, often the variability of a single point measurement,

$$
R O V=\frac{\sigma\left(\Delta k_{c_{\Delta t}}\right)_{\text {actual }}}{\sigma\left(\Delta k_{c_{\Delta t}}\right)_{\text {reference }}} .
$$

\section{A. Characterizing variability}

Various studies have applied the standard deviation of clear sky ramp rates and the relative output variability metrics to characterize variability. In general, studies show that longer time intervals correspond to larger ramp rates and higher variability (i.e., it is more probable to observe a large change in clear-sky index over a $1 \mathrm{~h}$ interval than over a one second interval). ${ }^{5,6}$ Studies also observe that longer temporal averaging periods lead to reduction in variability for a fixed time interval. ${ }^{5}$ This may be exacerbated when considering PV power production rather than solar irradiance because short-timescale excursions may result in inverter clipping, but are hidden in averaged solar irradiance time series. This leads to an overprediction bias in simulations that rely on coarse temporal resolution (e.g., hourly) data. ${ }^{7,8}$ Studies have also used schemes to classify high- and low-variability time periods using variability metrics based on both the clear-sky index $^{9}$ and direct solar irradiance. ${ }^{10}$

The spatiotemporal nature of variability has been characterized by a number of studies. Chiefly, attempts have been made to understand time series correlations between pairs of sites, and descriptions of variability are framed within that context. It has been proposed that any correlation between site pairs is primarily the result of shared cloud cover. ${ }^{3}$ It is generally well known that correlation between individual site pairs decreases with increasing site separation. Many studies have observed anisotropic correlation effects in the along-wind and cross-wind directions, ${ }^{11,12}$ which highlights the importance of cloud transport (advection) in determining the relationship between sites. Models have been developed to attempt to predict the degree of correlation between these site pairs, including anisotropic effects. ${ }^{13,14}$ Some of these studies have explicitly noted the importance of accounting for delayed cross correlation, which is observed to increase the pairwise correlation in the along-wind direction. ${ }^{11,13}$ One study by Al-Hilfi et al. ${ }^{15}$ directly compares the results from several of the published spatiotemporal correlation models. Using a slightly different approach, Klima and $\mathrm{Apt}^{16}$ describe the variability using power spectral density (PSD) of the time series and observe that larger plants have a steeper drop-off in spectral density, leading to a greater smoothing effect at high frequency (i.e., short time scales).

Efforts to characterize spatiotemporal variability can naturally be extended to the closely related tasks of spatially interpolating a sparse solar irradiance distribution (or an irregular distribution) and forecasting the spatial solar irradiance. Several different types of approaches have been used for these tasks. Lipperheide et al. ${ }^{17}$ utilize persistence of a known distribution of power production with respect to cloud advection to forecast future production for a plant. Similar assumptions about advection were used by Wang et al. ${ }^{2}$ to compute limits on the possible ramp rates that a plant could experience. Some studies have demonstrated various techniques for downscaling/interpolating solar irradiance, including by using kriging. ${ }^{18}$ Kriging that includes temporal information has also been used for forecast purposes, including the effects of cloud movement. ${ }^{19,20}$ Trained neural networks have also been demonstrated for spatial forecast purposes. ${ }^{21}$ Other approaches have looked at generating representative spatially distributed solar irradiance data using statistical techniques, ${ }^{22,23}$ a cupola, ${ }^{24}$ and simulation of cloud fields. ${ }^{14,25,26}$

\section{B. Modeling aggregation}

The studies that are most relevant to the present work are those that attempt to model the reduction in variability induced by spatial aggregation. An early attempt at this was that by Hoff and Perez, ${ }^{3}$ who utilized a combined model that considered the transition between cloud advection-dominated conditions and spatially decorrelated conditions in describing reduction in variability. By advection dominated conditions, we mean those for which a constant cloud speed and direction (i.e., relatively short time windows) lead to a very high spatial correlation across a plant when accounting for time lag. Hoff and Perez defined the dispersion factor, $D$, to distinguish these variability regimes based on the geographic density of the plant distribution. The dispersion factor is defined as the ratio of the plant's overall length, $L$, to the product of cloud transit velocity, $V_{c}$, and the sampling period of the measurement, $t_{\text {samp }}$,

$$
D=\frac{L}{V_{c} \cdot t_{\text {samp }}} .
$$

One limitation of the Hoff and Perez ${ }^{3}$ approach, as noted by the authors, was that it did not generalize for arbitrary distributions of generation, but instead limited itself to a uniformly distributed plant. Other approaches also leveraged the concept of determining variability reduction by loss of correlation in distributed site pairs, including those by Lave et al., ${ }^{27}$ Widen, ${ }^{28}$ and Elsinga. ${ }^{29}$ Further work by AlHilfi et al. ${ }^{30}$ utilized a combination of models to improve the overall model accuracy.

The wavelet variability model (WVM) by Lave et $a .^{27}$ is a broadly validated model ${ }^{31}$ and benefits from the availability of software implementations, such as that in pvlib-python. ${ }^{32}$ This model represents an arbitrarily distributed plant as a series of discretized points. In order to represent the geographic smoothing, it selectively filters the 
clear-sky index time series of a central reference node at different wavelet modes (essentially frequency bands). The WVM relies on the modeled pairwise correlation coefficients between the discrete sites, $\rho$, which in turn depend on the site separation distances, $L$, the cloud speed and the time interval, which in this case corresponds to the wavelet scale,

$$
\rho=\exp \left(-\frac{L}{\left(V_{c} / 2\right) \Delta t}\right) .
$$

The WVM scales the magnitude of each wavelet mode using a variability reduction index that is based on the sum of all possible pairwise correlation coefficients, as in Eq. (5). Cloud speed can be assumed to remain approximately constant relative to seconds- or minutesscale variability (e.g., 3-h cloud speed data were used by Lave and Kleissl $^{33}$ ). Thus, because the site pair spacing is constant, the scaling described by the WVM is essentially fixed in time,

$$
w_{P}(t)=\frac{w_{\text {ref }}(t)}{\sqrt{\frac{N^{2}}{\rho_{\text {sum }}}} .}
$$

A different approach by Marcos et al. ${ }^{34,35}$ represents the smoothing effect of a plant as a transfer function (TF) relative to a single measurement point input. The transfer function is empirically derived and is based on the form of a single pole low pass filter. The cutoff frequency was found to depend on the size of the plant in hectares. As such, it does not account for short-term changes to the cloud speed, but instead is tuned for long term variability. The model was validated against several metrics of longterm variability with success.

The existing models leave a gap in knowledge regarding spatial aggregation of solar irradiance. Hoff and Perez ${ }^{3}$ describe a continuum of conditions that can be observed in site-pair correlations, beginning with high site-to-site correlation due to frozen cloud advection and extending to full decorrelation between site pairs. Both the WVM and Marcos et al. ${ }^{35}$ approaches are well validated for representing longterm trends in variability, but are unable to resolve the role of cloud advection on short time scales. However, several possible characteristics of variability that are described in literature suggest that cloud advection plays a role. For example, in a validation of the WVM, Dyreson et al. $^{31}$ noted that the WVM predicts lower variability on short timescales (high frequency) than that observed in actual data. One possible explanation for this is that the WVM may be under predicting site-pair correlation, which is enhanced during cloud advection. Additionally, some studies ${ }^{11,29}$ observe anti-correlation in time series at certain along-wind site-pair distances, which is suggestive of a condition where periodically advecting clouds could cause sites to appear to vary in an out-of-phase manner.

In the present study, we attempt to determine whether cloud advection plays a role in real examples of spatial aggregation of irradiance and to describe how advection dominated conditions affect variability. We derive a model that obeys the physics of frozen cloud advection described by Hoff and Perez. ${ }^{3}$ Our model builds upon that by Hoff and Perez by representing a generalized plant shape. We adopt transfer functions (as suggested by Marcos et al. ${ }^{34}$ ) as a useful method to describe the frequency dependence of the smoothing of variability.

\section{CLOUD ADVECTION MODEL FORMULATION}

The following model development was first presented in a conference paper, ${ }^{36}$ but is reproduced here for completeness. The fundamental basis for this model is the assumption of frozen clouds that advect across distributed plant ${ }^{3}$ at a constant speed. A sketch of this behavior is shown in Fig. 1. For these purposes, advection is considered to be a 1-D effect; at any instant, clouds are all transported at a fixed rate, in a fixed direction, and do not evolve with time. The plant is represented as one-dimensional in space, which essentially assumes that the clouds move in a uniform front perpendicular to the direction of their motion. Through the following derivation, we will construct a representation of the variability of the 1-D distributed plant relative to that of a single point reference measurement.

We define the localized density of generation per unit distance as $d(x)$. Thus, the time series for the plant's aggregate solar irradiance, $p(t)$, is the product of this distribution and the local solar irradiance, $g(x, t)$, integrated over the plant's spatial extent, $X$,

$$
p(t)=\int_{X} d(x) \cdot g(x, t) d x .
$$

For 1-D advection of frozen clouds at a fixed speed, we recognize that the spatial variation of solar irradiance is actually based upon delay; any clouds that affect irradiance at the reference point have the same effect at each other location during their transit. In this case, solar irradiance at any point in the 1-D domain can be described as the solar irradiance at the reference point, delayed (or advanced) by an advective time, $\tau_{d}=\frac{x}{V_{c}}$. This assumes that the clear sky irradiance is approximately constant, necessitating that the transit time across the plant be relatively short. With these assumptions, the spatially distributed solar irradiance becomes

$$
g(x, t)=g_{r e f}\left(t-\frac{x}{V_{c}}\right) .
$$

The spatial coordinate, $x$, can be replaced by the delay coordinate, so that we rewrite the plant distribution as $d^{*}\left(\tau_{d}\right)$. Note that $d^{*}\left(\tau_{d}\right)$ should be normalized to have an integral of 1 , so that the transfer function has a magnitude of 1 at zero frequency.

When substituting $d^{*}\left(\tau_{d}\right)$, the aggregate plant solar irradiance becomes the convolution of the delayed solar irradiance with the plant, ${ }^{28}$

$$
p(t)=\int_{\frac{X}{V_{c}}} d^{*}\left(\tau_{d}\right) \cdot g_{r e f}\left(t-\tau_{d}\right) d \tau_{d}
$$

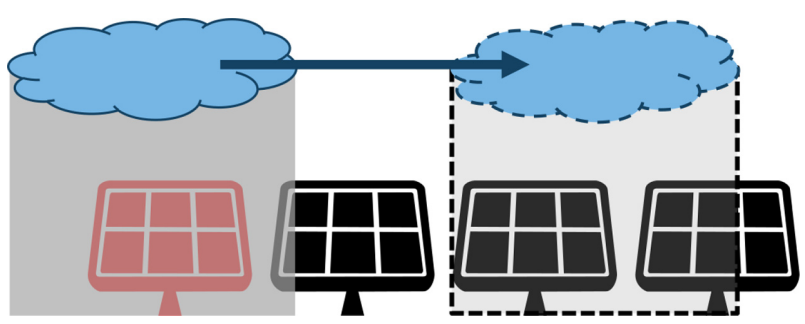

FIG. 1. Sketch of the shading effect as a cloud advects over a distributed plant between two instants in time. Aggregation refers to the plant summing up the distributed irradiance. The portion of the plant shown in red represents a reference measurement made at the upwind edge of the plant. 
Following the approach of Marcos et al., ${ }^{34}$ we represent the aggregation using a transfer function, $T F$, between the reference site solar irradiance and that of the plant. A transfer function is a common, frequency dependent representation of a system as a response (output) to a given input. In this case, the output is the plant's aggregated irradiance, $P$, while the input is the single point reference measurement, $G_{r e}$, leading to the transfer function relationship given by the following equation:

$$
T F(f)=\frac{P(f)}{G_{r e f}(f)} .
$$

Because time domain convolution is equivalent to frequency domain multiplication, the transfer function simply reduces to the Fourier transform of the plant's spatial distribution,

$$
T F(f)=\frac{D^{*}(f) \cdot G_{r e f}(f)}{G_{r e f}(f)}=D^{*}(f)=\mathscr{F}\left[d^{*}\left(\frac{x}{V_{c}}\right)\right] .
$$

The position of the reference sensor within the plant distribution can be accounted for by adding or subtracting time delay from the transfer function based on the cloud transit time from the upwind edge of the plant to the reference sensor. Mathematically, this operation consists of rotating the phase of the transfer function by an angle proportional to the frequency as in Eq. (11). Note that for a nonuniform plant, the effective center of the plant is basically aligned with the centroid of the distribution $d(x),{ }^{37}$

$$
T F_{\text {rot }}=T F \cdot \exp \left(2 \pi i f \frac{\Delta x}{V_{c}}\right) .
$$

This formulation allows computation of the transfer function based only on the plant's 1-D spatial distribution and the cloud speed. Thus, for a given plant and cloud speed condition, we could compute a predicted $P(f)$ and use the inverse Fourier transform to compute the plant's aggregate time series, $p(t)$.

\section{A. Calculation overview}

The steps required to compute the smoothed time series using the transfer function are summarized here.

\section{Computing the plant transfer function}

(1) Gather the input data:

- Cloud speed and direction.

- Plant layout including reference location.

(2) Represent plant distribution as a numerical 1-D array, $d^{*}\left(\tau_{d}\right)$, in the cloud motion direction.

(3) Normalize to ensure that $d^{*}\left(\tau_{d}\right)$ has an integral of 1 .

(4) Compute the fast Fourier transform (FFT) of $d^{*}\left(\tau_{d}\right)$ to obtain $T F(f)$.

- If necessary, first pad $d^{*}\left(\tau_{d}\right)$ with trailing zeros to increase frequency resolution.

(5) Apply Eq. (11) to account for position of the reference within the plant, yielding $T F_{\text {rot }}(f)$.

\section{Computing a smoothed time series}

(1) Perform FFT on the reference time series to obtain $G_{r e f}(f)$.
(2) Multiply $G_{r e f}(f)$ by the transfer function, $T F_{\text {rot }}(f)$, to obtain filtered output, $P(f)$.

- Interpolation may be necessary to ensure that both frequency axes match. Use care because both quantities are complex numbers.

(3) Perform inverse FFT to yield the filtered irradiance time series, $p(t)$.

(4) If desired, divide by the clear sky irradiance to compute $k_{c}(t)$.

\section{B. Model results for simplified case}

It may be instructive to first consider a simple case to highlight the model's behavior. For example, we may consider the smoothing caused by a uniformly distributed 1-D plant of length $L_{p}$, relative to a sensor placed at its upwind edge. This plant can be thought of numerically as an array of numbers, having the value of 1 within the plant footprint and zero elsewhere,

$$
d^{*}\left(\tau_{d}\right)= \begin{cases}1, & 0 \leq x \leq L_{p}, \\ 0, & x>L_{p} .\end{cases}
$$

The transfer function for this distribution (i.e., its FFT) corresponds to a sinc filter, as seen in Fig. 2. We may learn a few basic facts about the way that this model behaves in general by inspection of the figure for this simple case.

\section{Magnitude response}

Overall, the transfer function magnitude decreases with increasing Strouhal number: $S t=\frac{f L_{p}}{V}$. Specifically, it decreases in proportion to $\frac{1}{S t}$, representing a low-pass filter character, which agrees with the smoothing we expect to observe. The Strouhal number is similar to the dispersion factor discussed by Hoff and Perez. ${ }^{3}$ However, one major difference is that here we use the length scale, $L_{p}$, to represent the entire size of the plant, rather than the plant density as in Hoff and Perez.

We also observe that the transfer function experiences periodic zero magnitude values, which correspond to integer values of the Strouhal number. Physically, the zero magnitude events visible in the transfer function reflect conditions where spatial destructive interference causes a zero net oscillating response for the whole plant. In the case of the first such occurrence, the cloud spacing is equivalent to the plant size, mathematically describing a sinusoidal distribution over the plant that is half cloudy, half clear. If we view the plant as upwind and downwind halves, they experience out-of-phase oscillations and together sum to zero.

\section{Phase response}

The phase of the transfer function exhibits rapid phase shifts of $180^{\circ}$ that occur at integer values of the Strouhal number, corresponding to zero-magnitude values. The dominant characteristic of the phase is a linear decline, indicating time delay. This is expected in that the "average" plant response occurs as a cloud passes over the center of the plant, while the reference input in this example was located on the upwind edge. The shift applied in Eq. (11) changes the amount of delay and effectively moves the reference site relative to the plant. This allows the model to account for a reference that is located anywhere. 

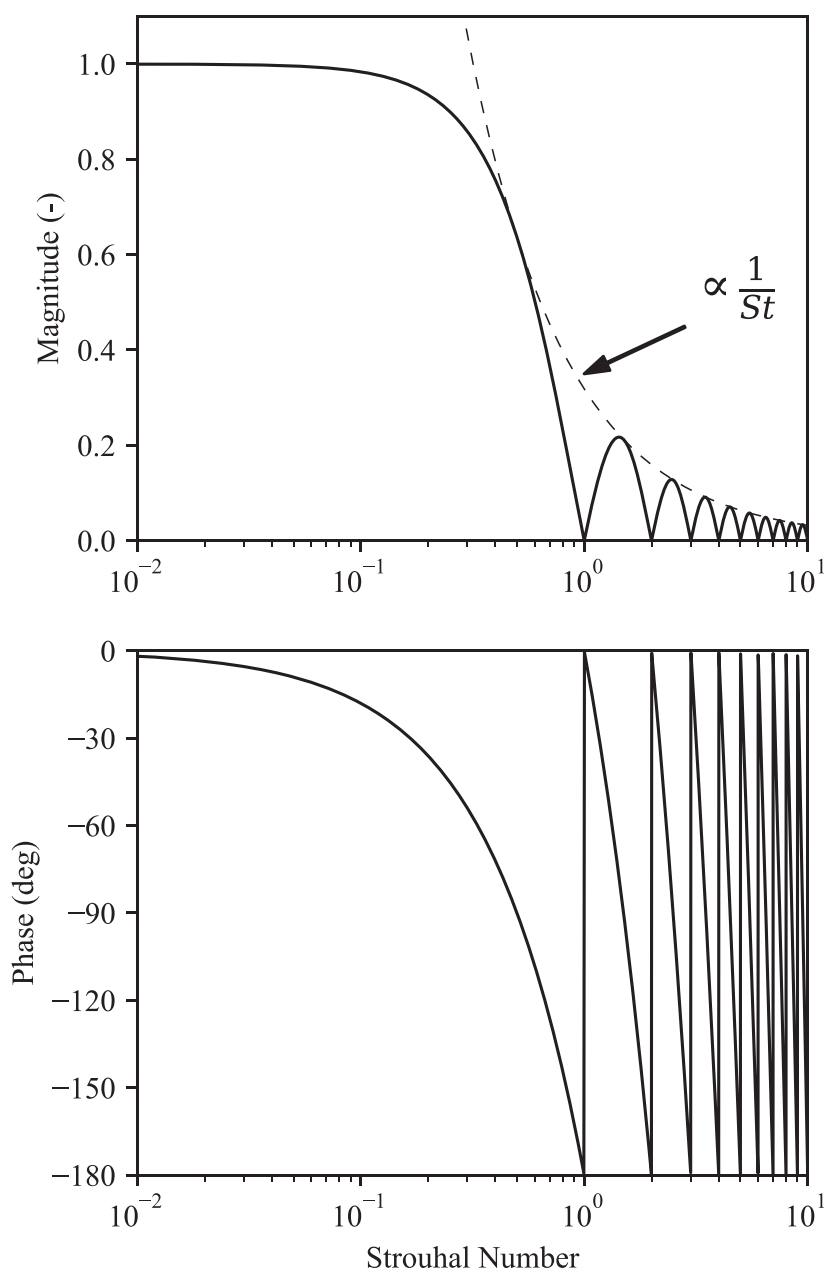

FIG. 2. Modeled transfer function for uniformly distributed plant. Frequency axis scaled to Strouhal number.

\section{Model description summary}

In summary, the cloud advection model (CAM) predicts a lowpass filter with time delay, which is consistent with the smoothing of spatiotemporal variability. The dynamic interference-like phenomena described in Sec. III B 1 occur in this model as an artifact of advection and would not be expected to occur for correlation-based models of variability. In order to determine whether advection is important in real irradiance data, we will apply the model against a real measurement dataset and investigate these and other characteristics of the transfer function.

\section{VALIDATION RESULTS AND DISCUSSION}

In order to investigate the importance of advective effects described by the model, we compared predictions of the transfer function with real data transfer functions between a single point measurement and a spatially distributed plant representation.

\section{A. Dataset}

We used data from the HOPE-Melpitz measurement campaign ${ }^{38}$ to make this comparison. The HOPE-Melpitz campaign was conducted outside of Melpitz, Germany. Global horizontal irradiance (GHI) was measured by 50 single-point sensors. The sample rate was $1 \mathrm{~Hz}$ and measurements lasted throughout the period from September 10-27, 2013. Some data were still available outside that period, but not for all sites. The overall measurement site footprint was approximately $3 \mathrm{~km}$ in the longitude direction by $2 \mathrm{~km}$ in the latitude direction. A dense, central cluster of 26 stations had a footprint of roughly 400 $\times 200 \mathrm{~m}^{2}$, with the remaining 24 stations more sparsely spaced in the larger bounding region. A satellite image with the layout of the 50 point measurement sites is shown in Fig. 3 .

Uncertainty and quantization error pose a potential source of error in interpreting the data. Madhavan et al. report overall uncertainties for the sensors of around $35-100 \mathrm{~W} / \mathrm{m}^{2}$ for the $1000 \mathrm{~W} / \mathrm{m}^{2}$ full-scale measurement. ${ }^{39}$ Although specific bin size is not reported, the analog-to-digital conversion system is reported by Madhavan et al. to have a resolution of 10 bits, which at full scale would correspond to a bin size around $1 \mathrm{~W} / \mathrm{m}^{2}$. Close inspection of the time series shows that quantization is evident during relatively steady solar irradiance conditions (e.g., cloud cover) and appears to have a bin size around $1.5 \mathrm{~W} / \mathrm{m}^{2}$. Thus, it is difficult to distinguish actual variability of this magnitude from measurement noise at high frequency.

\section{B. One-dimensional validation with manually identified advection}

We first attempted to validate the model predictions against a one-dimensional subset of measurement sites aligned with cloud advection. We manually identified the period of September 8, 2013 from 09:15 to 10:15 AM from the time series. During this period, clouds were observed to advect in a south-to-north direction. We selected a subset of measurement sites aligned with the cloud motion and passing through the center of the measurement footprint. One site (number 28) was excluded due to the fact that its output was

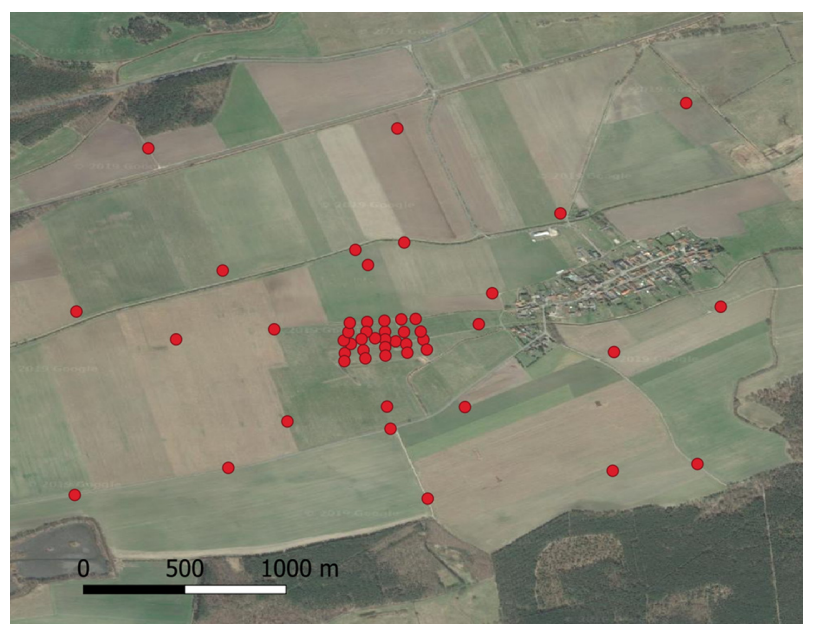

FIG. 3. HOPE-Melpitz measurement campaign site layout with 50 individual stations. Central station (ID No. 40) is located at $51.52566^{\circ} \mathrm{N}, 12.927199^{\circ} \mathrm{E}$. 
inconsistent with advection. The advective speed was identified by comparing the lagging cross correlation between the aligned sites, and a cloud velocity of $20 \mathrm{~m} / \mathrm{s}$ from south to north was observed. The time series for the selected subset of points are shown in Fig. 4.

In order to apply the model, each of the sites' positions was projected onto the north-south direction, yielding the plant distribution shown in Fig. 5. The modeled transfer function for this distribution was calculated using the FFT, as described in Sec. III.

To calculate the frequency representation of the real data, the time series for each site were normalized to have the same mean solar irradiance as the reference. The sites were then aggregated to represent the plant, and the time series were transformed to the frequency domain by an FFT. The reference site time series was also transformed by an FFT.

\section{Power spectra}

The power spectral density (PSD), or power spectrum, can be used to represent how the power contained by a time signal is split up among various frequency components. Figure 6 shows the difference in power spectra between the reference and aggregate plant time series. Compared to the reference, the magnitude of the aggregate plant is significantly reduced for frequencies corresponding to periods between roughly $5 \mathrm{~s}$ and $5 \mathrm{~min}$. The overall decrease in power spectrum magnitude with increasing frequency is well described in the literature. The extremely low magnitudes observed beyond roughly $0.1 \mathrm{~Hz}$ (tens period) are smaller than the overall bin resolution of the data acquisition system, and interpretation in this range should be made with care due to the potential influence of quantization error, as described previously.

\section{Transfer functions}

The transfer function between the reference site and the aggregate plant was computed as the ratio between the aggregate and reference frequency representations. We compared the CAM predictions

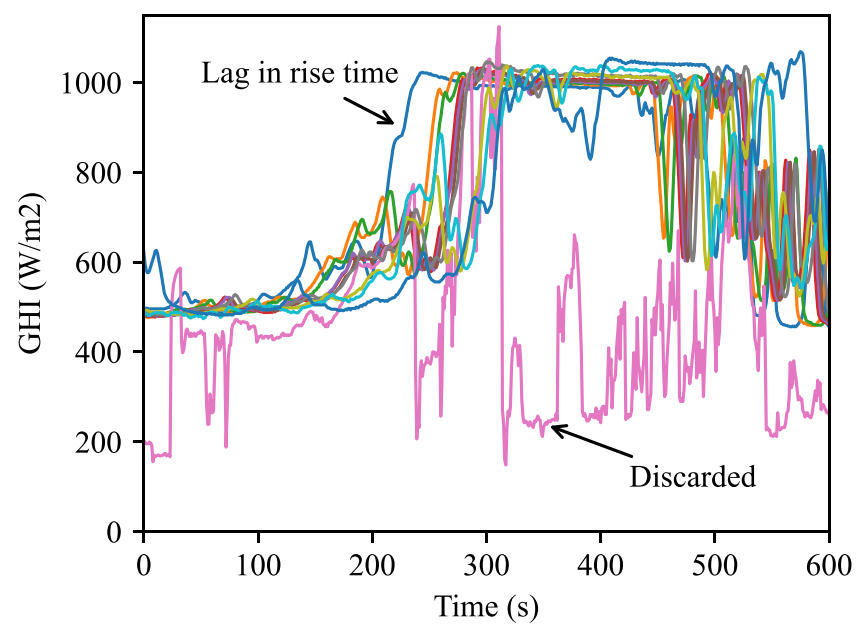

FIG. 4. Individual site time series for the plant subset used in the 1-D validation, demonstrating advection as identified via lag. The time series for the site discarded for $\mathrm{QC}$ is labeled.
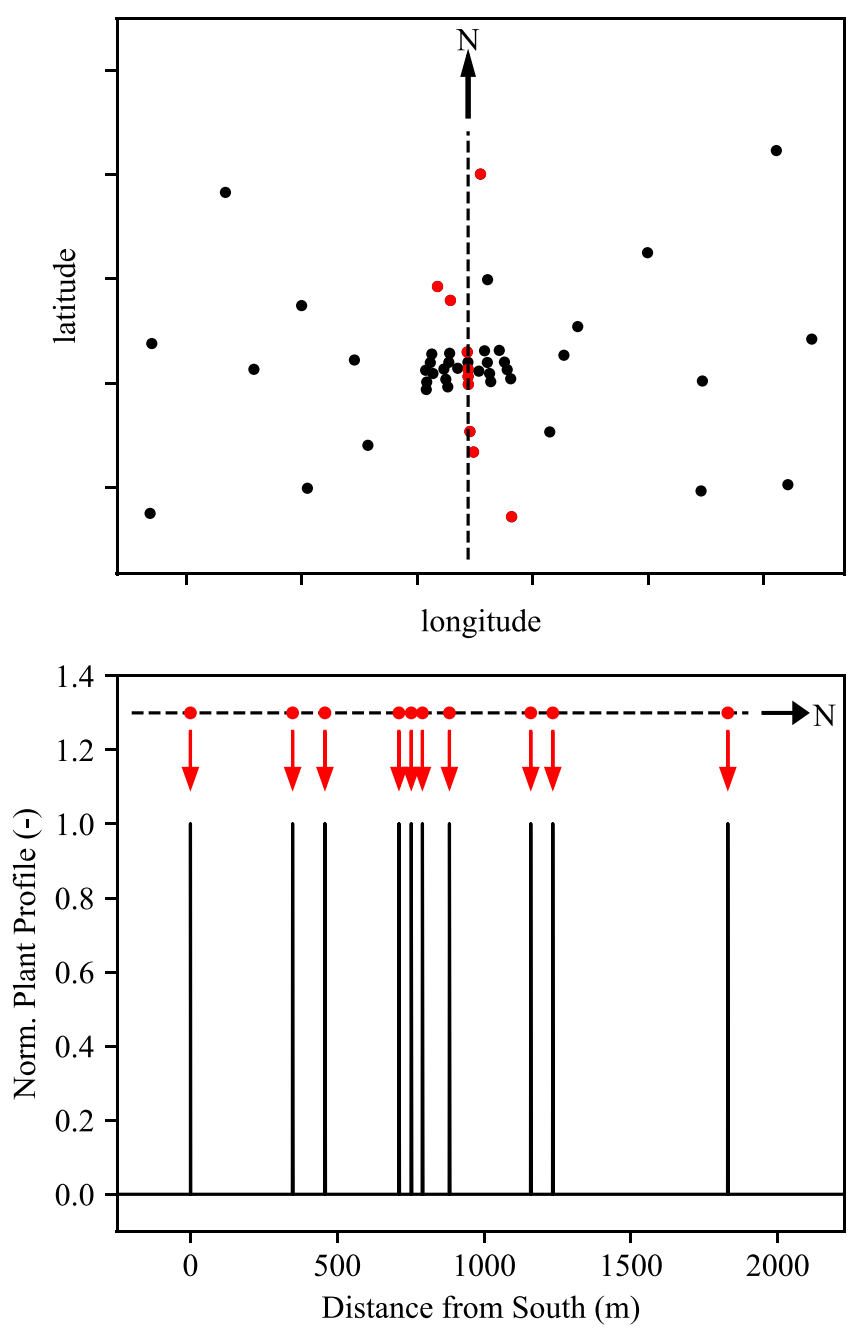

FIG. 5. (top) Subset of points used for the one dimensional north-south aligned case. The red points are projected perpendicularly onto the north-south cloud transit direction, shown by the dotted line, to form the plant distribution. (bottom) The plant distribution, $d(x)$, for the projected measurement sites. Because the singlepoint sensors are infinitesimally small, the plant's spatial distribution essentially consists of a sum of Kronecker delta functions with each centered at one of the highlighted measurement locations. As each sensor is equivalent, the magnitude of the distribution is normalized.

with the WVM by Lave et al. ${ }^{27}$ and the model of Marcos et al. ${ }^{35}$ (subsequently referred to as the Marcos model). The cutoff frequency of the Marcos model was manually tuned to match the cutoff frequency of the actual data due to the fact that the analytical pole expression given by Marcos et al..$^{35}$ relies on a spatial area, which is not suitable for a 1-D plant distribution. The comparison of transfer functions is shown in Fig. 7.

With reasonable accuracy, all three models predict the overall low-pass filter shape of the transfer function, along with the cutoff frequency. However, several unique features of the data are predicted only by the cloud advection model (CAM). Specifically, the magnitude 


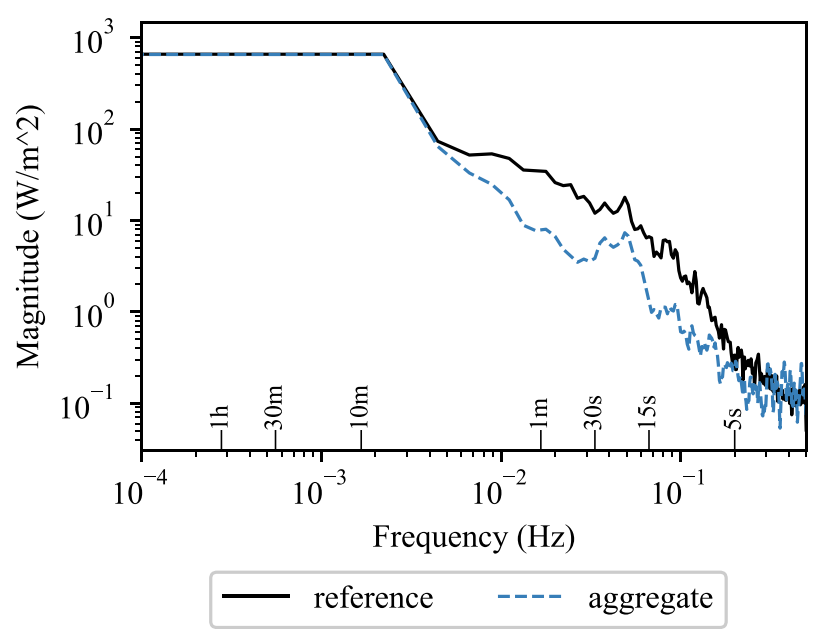

FIG. 6. Power spectra of the reference site and aggregate site subset described in Fig. 5 for the selected time span described in Sec. IV B. Power spectrum is scaled such that magnitude represents the amplitude of oscillation at the given frequency. Note that frequency resolution (effect visible, e.g., at low frequencies) is limited by the $1 \mathrm{~h}$ window. Spectra are smoothed using eight averages with a Hanning window and $50 \%$ overlap.

rebound that occurs around a frequency of $0.05 \mathrm{~Hz}$ is only predicted by the CAM. This dynamic feature is caused by spatial interference patterns associated with cloud motion over the plant, as described previously in Sec. III B. Additionally, the real transfer function exhibits complexities in the phase dynamics that are only predicted to occur by the CAM. The phase of the WVM is essentially constant due to its implicit assumption of a centrally located reference site. The Marcos model predicts the phase behavior expected for its single-pole filter basis.

The match between the real data and the CAM's prediction worsens with increasing frequency (beginning around $0.1 \mathrm{~Hz}$ ). This can be explained by considering the loss of coherence in the real data transfer function. The sharp decline in coherence beyond a frequency of around $0.1 \mathrm{~Hz}$ indicates that oscillations in the reference and aggregate plant signals are no longer well correlated at these frequencies. This may indicate a failure of the frozen clouds assumption on short time and length scales, or it may indicate a source of uncorrelated noise in the data. Due to the declining overall magnitude of the power spectrum at these frequencies, it is difficult to differentiate these effects with the present data.

We also apply the CAM to predict the smoothing of the solar irradiance time series, including the effect of a variable reference site position. Three time series predictions for reference sites situated on the north, south, and center of the distribution are shown in Fig. 8. As expected, the spatial distribution of generation produces a smoothed time series relative to the reference, which matches with the low-pass filter behavior at play. As each case uses the same plant distribution, the aggregate time series is exactly identical in each case. However, the reference site time series is advanced or delayed relative to the aggregate time series according to its upwind or downwind position. The CAM is able to produce a reasonable approximation of the aggregate time series based on each of the three reference site choices.
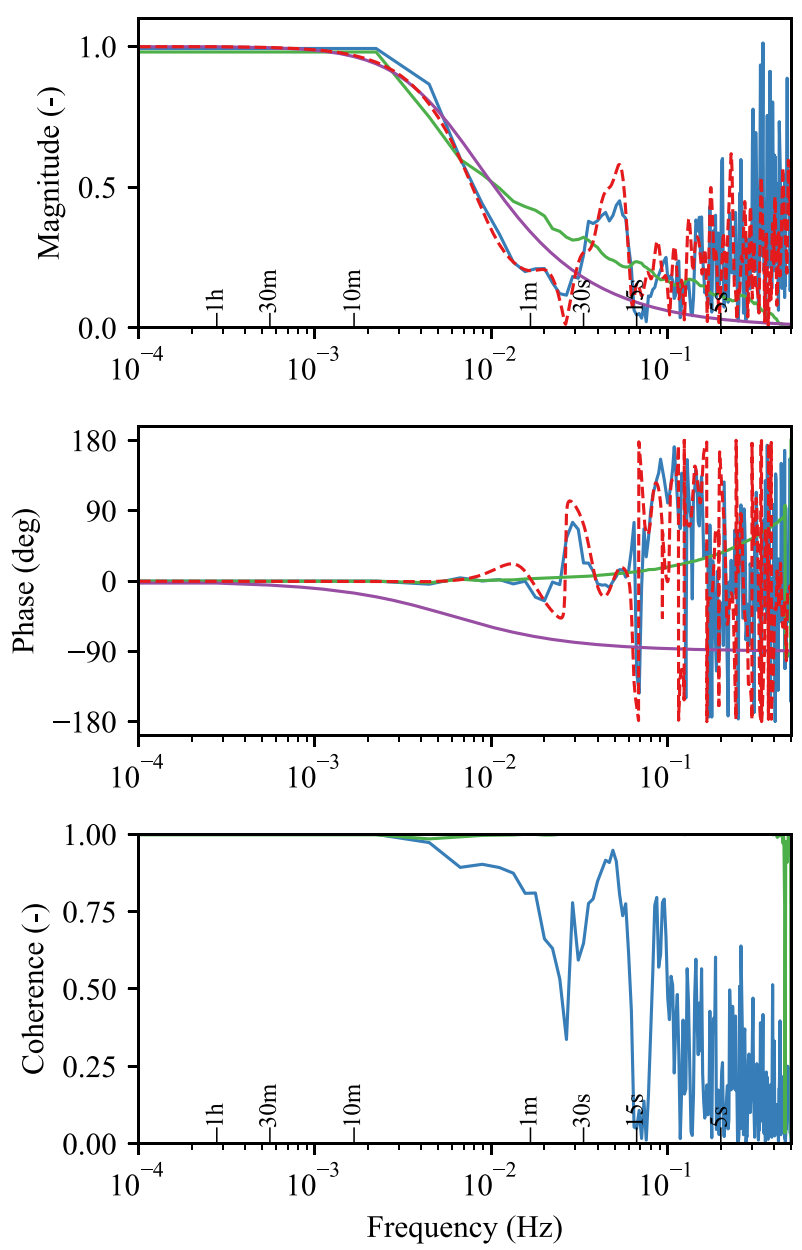

$\longrightarrow$ real $\longrightarrow$ WVM $\longrightarrow$ Marcos ---- CAM

FIG. 7. Transfer function for the reference and aggregate sites described in Fig. 5 for the selected time span described in Sec. IV B. Predicted transfer functions for several models are compared. Real and WVM transfer functions are smoothed using eight averages with a Hanning window and $50 \%$ overlap.

We may also consider how the transfer function changes with changes in the plant layout. Figure 9 shows transfer function results and model predictions for four separate plant configurations. Changing the plant configuration substantially alters the shape of the transfer function. Notably, a distribution of the overall plant over a larger spatial extent creates a transfer function with a lower cutoff frequency. This is consistent with the Strouhal number scaling described in Sec. III B 1. Besides the overall low-pass filter shape, each case in Fig. 9 exhibits different dynamic characteristics. The fact that these changing dynamic characteristics are predicted by the CAM but not the WVM and Marcos model suggests that these dynamics are a direct result of coherent cloud advection. Thus, in order to accurately represent the smoothing of the time series, it is important to account for advection in this case. 

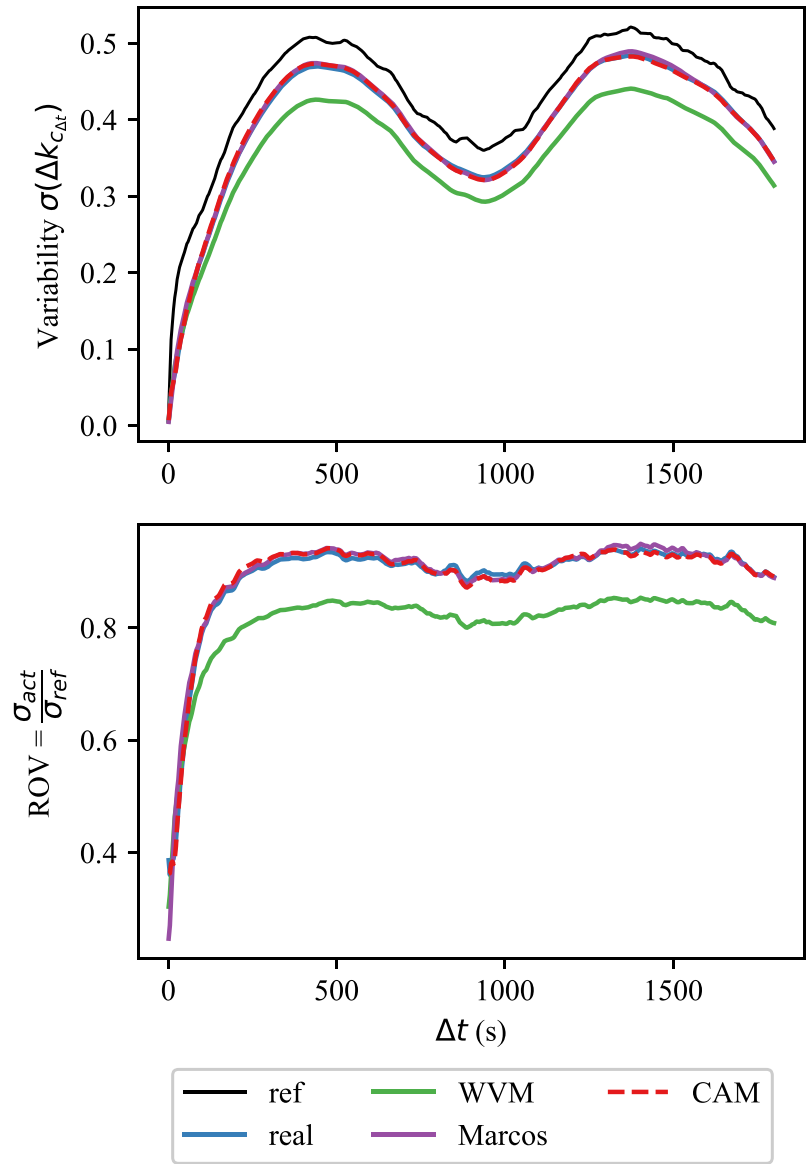

FIG. 8. Modeled and actual time series for three separate reference sites. Cloud motion direction is south-to-north.

The importance of advection is emphasized when considering the root mean square error $\left(\mathrm{RMSE}_{k_{c}}\right)$ in the prediction of the clear-sky index time series by each model, given in Table I. The CAM produces a better prediction of the time series for each of the four plant configurations.

\section{Variability}

We also compared the ability of the model to predict variability according to traditional metrics, such as $\Delta k_{c}$ and $R O V$. Figure 10 shows a comparison of these metrics for each of the three models. Figure 10 shows that the plant uniformly reduces the variability for all time intervals. The CAM accurately represents this reduction for the each interval according to these metrics. The WVM overpredicts the degree of variability reduction for this advection-dominated condition. The Marcos model yields a comparable accuracy to the CAM, bearing in mind the fact that the Marcos cutoff frequency was manually adjusted to match that of the actual transfer function for these data. While we can infer some information about the performance of the CAM from these results, it is possible for multiple time series representations to produce the same ramp rate variance. ${ }^{22,23}$ Thus, it is better
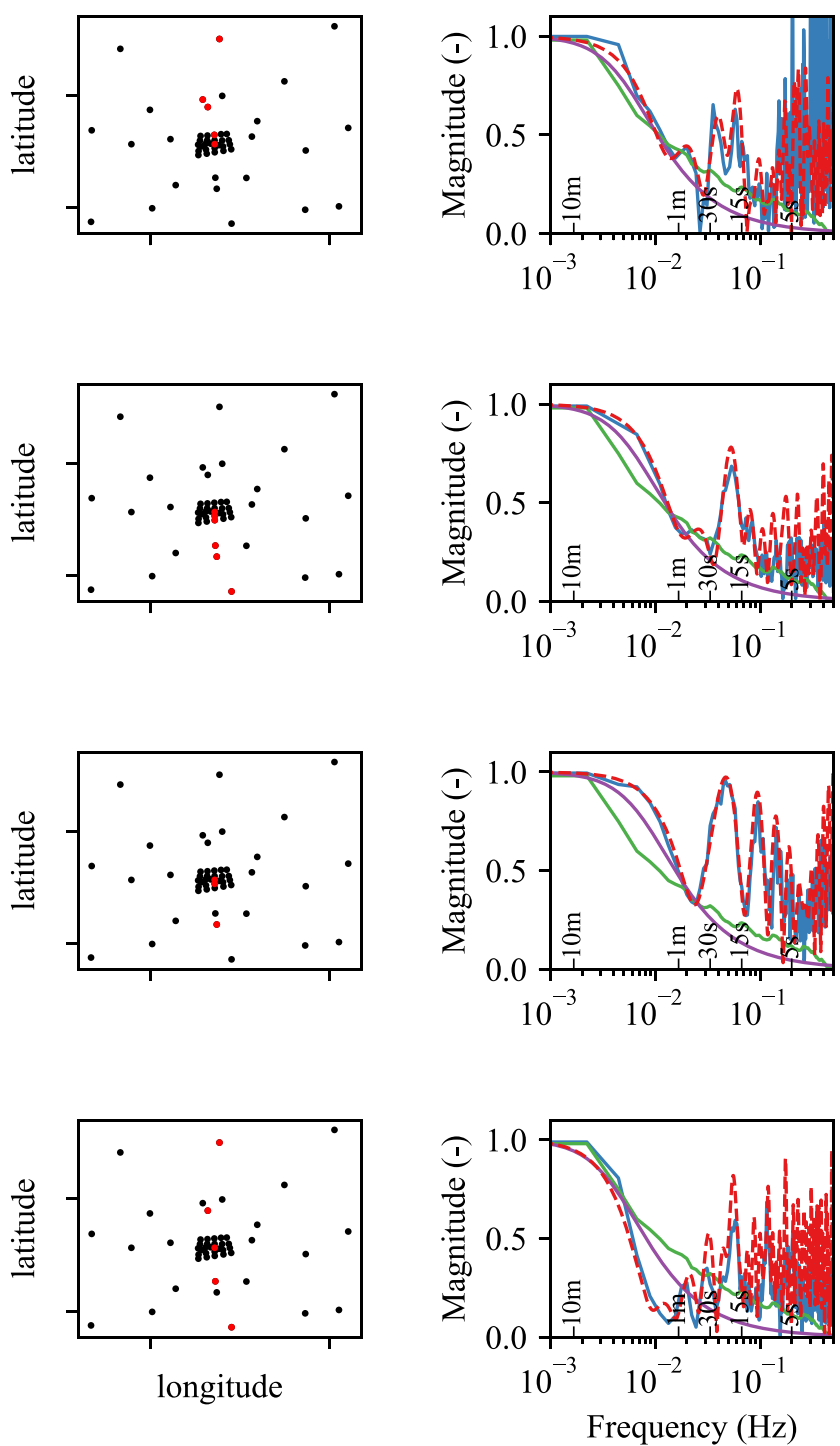

real $\longrightarrow$ WVM

Marcos ---- CAM

FIG. 9. Transfer function comparison for the various models with respect to four separate variable plant configurations. Reference sites selected for each case are highlighted in the left column. Real and WVM transfer functions are smoothed using eight averages with a Hanning window and $50 \%$ overlap.

to rely on the comparisons of the transfer function or time series RMSE to compare model performance, as in Sec. IV B 2.

\section{Identifying cloud speed and considering general advection}

In addition to the manually identified advection-dominated condition described in Sec. IV A and IV B, we wish to analyze the performance of the model with respect to variable cloud motion direction and speed. Methods are described in literature for the identification of 
TABLE I. RMSE of the clear-sky index time series relative to the real aggregate data for the plant configurations shown in each row of Fig. 9.

\begin{tabular}{lccc}
\hline \hline Row & CAM & WVM & Marcos \\
\hline 1 & 0.039 & 0.083 & 0.048 \\
2 & 0.028 & 0.067 & 0.133 \\
3 & 0.020 & 0.064 & 0.118 \\
4 & 0.038 & 0.054 & 0.088 \\
\hline \hline
\end{tabular}

cloud motion from multi-site datasets, such as the HOPE-Melpitz data used here. Cross spectral analysis (CSA) is one example of a method for identifying cloud motion vectors. It was first proposed by Inoue et $a .^{40}$ and Shinozaki et al., ${ }^{18}$ and was later modified by Jamaly and Kleissl. ${ }^{41}$ Another method, the cross correlation method (CCM), uses cross correlation between successive snapshots of solar irradiance and
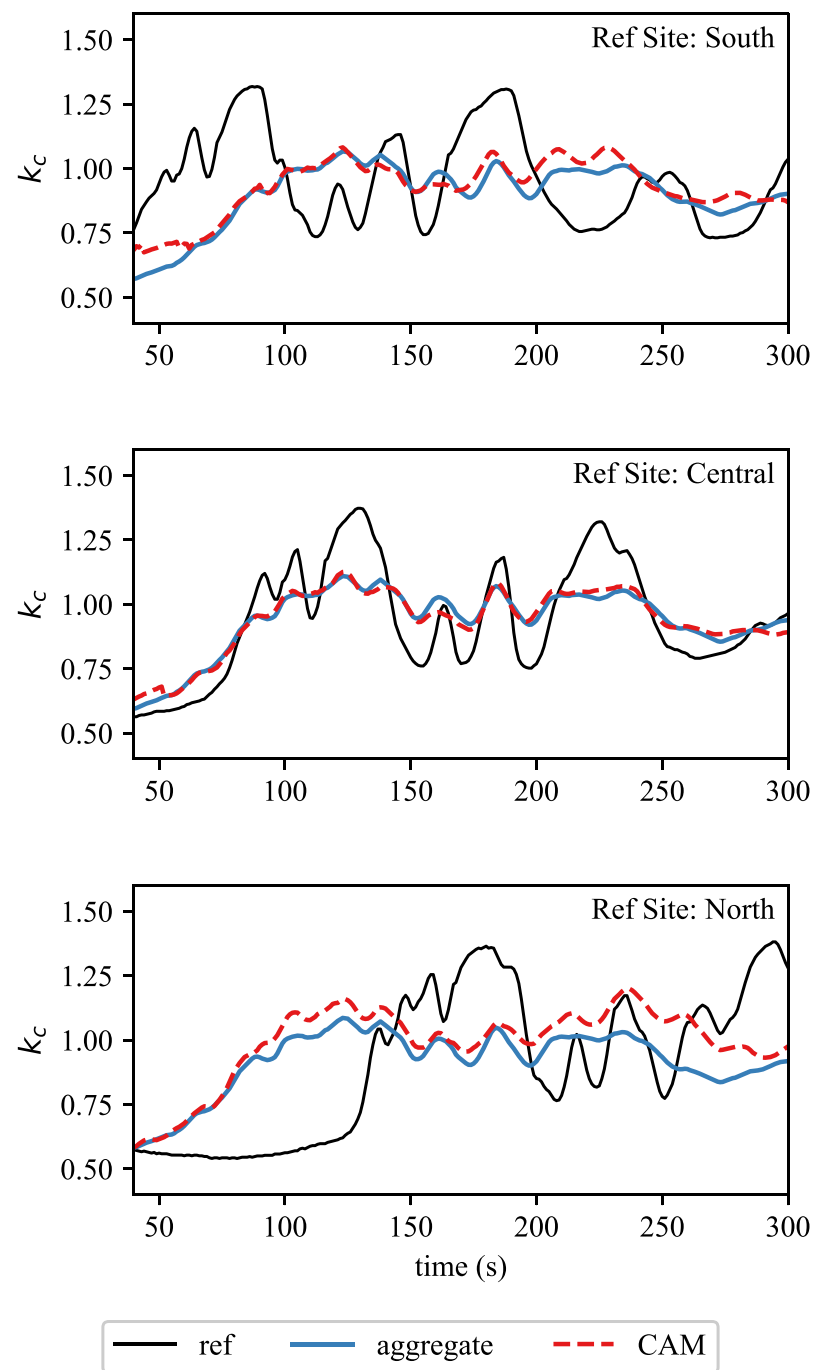

FIG. 10. Variability comparison between different model approaches. was described in the literature by Jamaly and Kleiss ${ }^{41}$ and EspinozaGavira et al. ${ }^{42}$ The CCM requires either that measurement data be gridded or that kriging (or a similar technique) be used to create a gridded dataset. ${ }^{42}$ As such, the modified form of the CSA described by Jamaly and Kleissl ${ }^{41}$ is easier to apply to the present dataset and was adopted to compute the cloud motion vectors in a general way.

The CSA method consists of adjustment of the angle of cloud motion such that it minimizes the variance in the calculated velocities for each site pair. Velocity is calculated by the ratio of the site separation, $r_{i}$, and the time delay for which the peak correlation between those two sites occurs, $\Delta T_{i}$. For the present scenario, where we wish to compute transfer functions based on a single reference, we limit the pairwise velocity computation to site pairs containing the reference. As in Jamaly and Kleissl, ${ }^{41}$ we minimize the variance of the computed velocity objective function $V_{\text {var }}$, relative to the wind direction as specified by the wind unit vector, $\hat{v}$,

$$
V_{v a r}(\hat{v})=\operatorname{var}\left(\frac{\vec{r}_{i} \cdot \hat{v}}{\Delta T_{i}}\right)
$$

Once an optimum cloud unit vector is identified, we compute the cloud speed as the median of the individual site pair velocities. We implemented the CSA method for each 1-h period within the full HOPE-Melpitz dataset. We adopted the quality control approaches proposed by Jamaly and Kleiss ${ }^{41}$ with a few modifications. We set $\max _{\text {lag }}$ to be the length of the time series and perform quality control to eliminate unsuitable site-pairs. We then perform the optimization on all remaining sites. We reject hours with $k_{c}<0.4$ as overcast conditions for which site-pair delay is unlikely to yield useful cloud motion information. We also reject periods for which there is very little difference between the maximum pair correlation at zero lag and the maximum lagging pair correlation, which helps to eliminate cases for which $\Delta T_{i}$ is unreliable. ${ }^{43}$

Once the cloud direction and speed have been computed, we construct the plant distribution, $d(x)$, by projecting site distance vectors onto the motion direction $\left(\overrightarrow{r_{i}} \cdot \hat{v}\right)$ for all sites that passed quality control. This collapses the two dimensional distribution of the plant into a one dimensional representation, along the cloud advection direction. Transfer functions are computed as discussed previously (refer to Sec. III A 1), and comparisons are made with the transfer function described by the real aggregate data.

In Fig. 11, we compare the transfer functions predicted by the model for several different wind conditions identified using the CSA method. Note that we omit the Marcos model from this comparison due to the requirement for manual adjustment of the cutoff frequency. As in previous examples, the transfer function always represents a low-pass filter. The high frequency advective dynamics are present for some cases; however, we observe that they are somewhat less significant than in the purely one-dimensional case. The dampening of these high frequency dynamics is caused by the denser spacing of sites that occurs as they are projected onto the wind-direction axis. The CAM provides a reasonable match for the data, considering both cutoff frequency and the dynamic behaviors that are visible.

We also compare the transfer functions when including all the available sites (i.e., even including those that failed quality control). These results are shown in Fig. 12. Given that the overall plant length is relatively constant in this case, we may more clearly observe the relationship between wind speed and the cutoff frequency of the transfer 

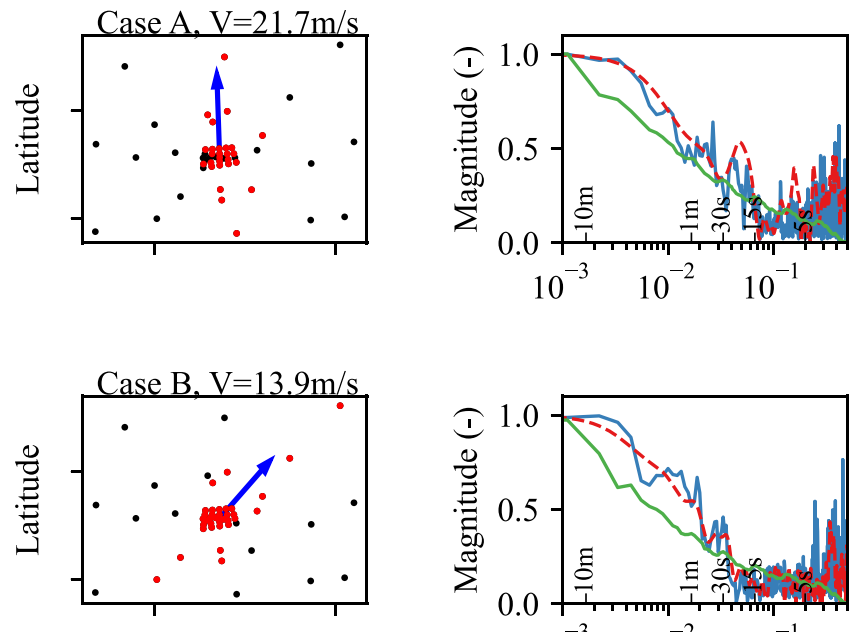

$10^{-3} 10^{-2} 10^{-1}$
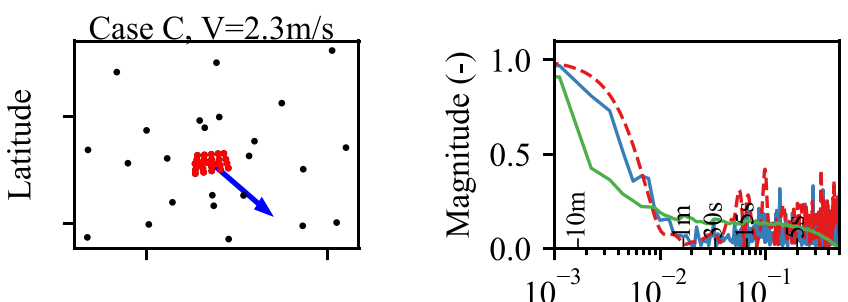

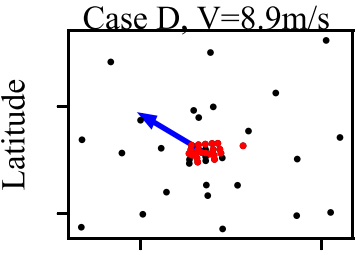

Longitude

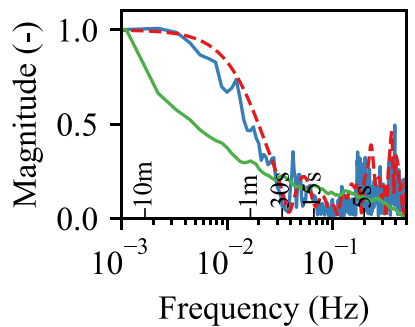

WVM
FIG. 11. Comparison of multiple cases for which the wind direction was identified systematically. Red sites are those that passed the quality control for wind speed determination and are the only sites used to compute the transfer function. Arrows show the cloud motion direction. Real transfer functions are smoothed using four averages with a Hanning window and $50 \%$ overlap.

function. Higher wind speeds correspond to higher cutoff frequencies and vice versa. In general, the inclusion of additional sites serves to dampen the high frequency dynamics of the transfer function, both in the real data and the model.

We also considered a few quantitative metrics to assess the performance of the models. We calculated the root mean square error $\left(\mathrm{RMSE}_{k_{c}}\right.$ ) for the predictions of the clear-sky index time series. Results for the four cases shown in Figs. 11 and 12 are given in Table II. The CAM outperforms the WVM uniformly for the cases where we only
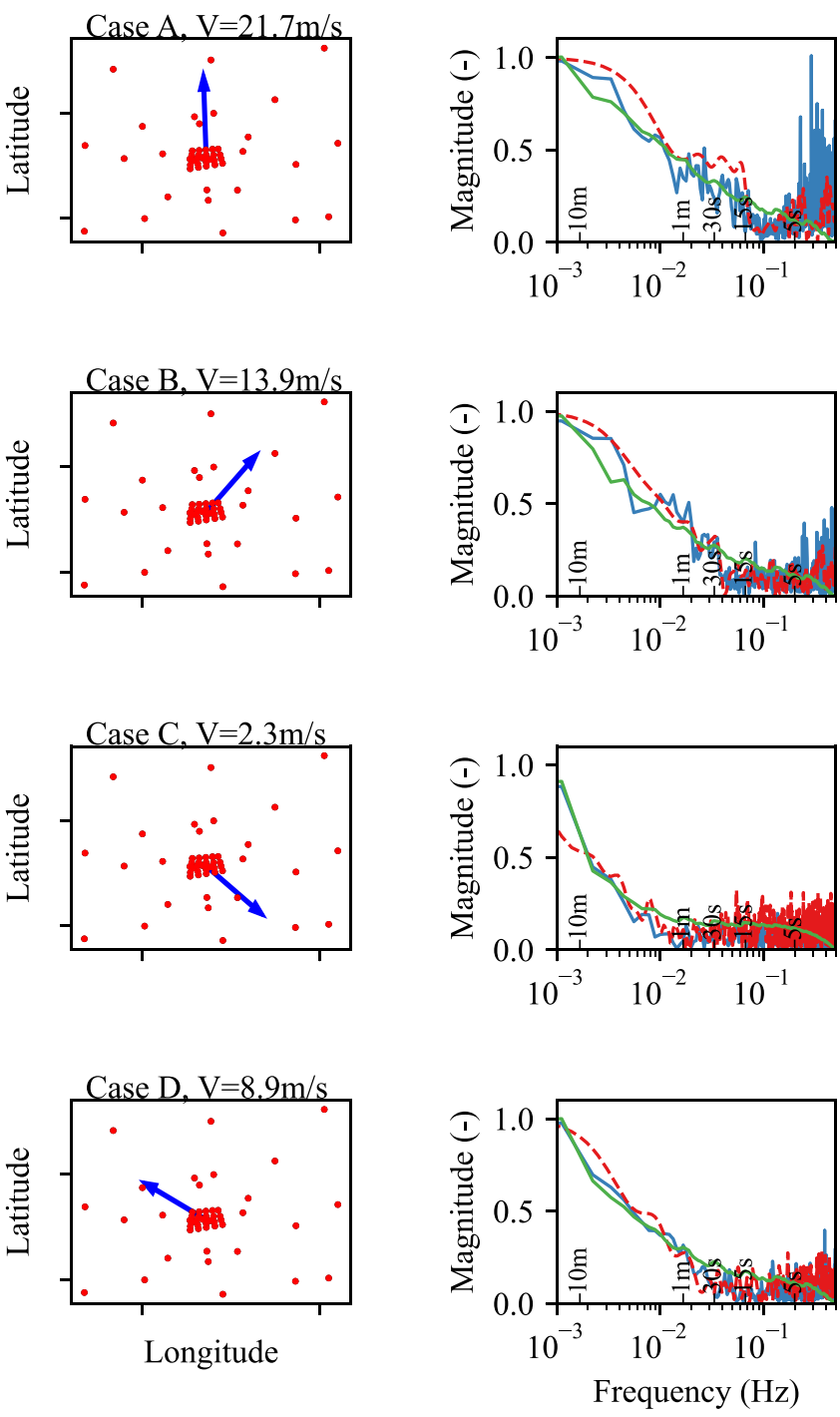

$\longrightarrow$ real ---- CAM $\longrightarrow$ WVM

FIG. 12. Comparison of multiple cases for which the wind direction was identified systematically. Time windows are the same as in Fig. 11. All sites are used in computation of the transfer function. Arrows show the cloud motion direction. Real transfer functions are smoothed using four averages with a Hanning window and $50 \%$ overlap.

consider the sites that pass quality control, while the WVM has superior performance when considering all measurement sites. This is an indication of the failure of the uniform frozen turbulence assumption that the CAM is based on. That is to say, the CAM assumes that when accounting for advective delay, the clear-sky index time series is stationary over the entire plant. The WVM, which was developed specifically to model the decorrelation between distributed sites, shows better performance in this case. This may indicate that decorrelation becomes more important than advection as a larger plant footprint is considered, specifically in the cross-wind direction. 
TABLE II. RMSE $k_{k_{c}}$ for clear-sky index time series predictions. Cases refer to the rows of Figs. 11 and 12.

\begin{tabular}{lcccc}
\hline \hline Case & $\begin{array}{c}\text { CAM } \\
\text { (QC only })\end{array}$ & $\begin{array}{c}\text { WVM } \\
\text { (QC only) }\end{array}$ & $\begin{array}{c}\text { CAM } \\
\text { (all sites) }\end{array}$ & $\begin{array}{c}\text { WVM } \\
\text { (all sites) }\end{array}$ \\
\hline A & 0.020 & 0.028 & 0.036 & 0.031 \\
B & 0.025 & 0.038 & 0.043 & 0.035 \\
C & 0.070 & 0.125 & 0.082 & 0.061 \\
D & 0.031 & 0.062 & 0.053 & 0.038 \\
\hline \hline
\end{tabular}

In addition to the four specific cases shown previously, we also consider the accuracy of the model across all possible time periods that allow a successful application of the CSA. In addition to computing the RMSE for all of these windows $\left(\mathrm{RMSE}_{k_{c}}\right)$, we also compute the RMSE of the model in predicting the transfer function cutoff frequency (measured at $50 \%$ of the low-frequency magnitude). We will label this quantity as $\mathrm{RMSE}_{f_{c}}$. These metrics are shown in Table III. Results uniformly follow those for the preceding analysis: the CAM performs better when limiting sites by quality control, while the WVM performs better when considering all sites. Scatter plots of the cutoff frequencies are also shown in Figs. 13 and 14. The CAM tends to overpredict the cutoff frequency for both the quality controlled and all-site cases. The WVM significantly underpredicts the cutoff frequency for the quality controlled case, which is consistent with our previous observation that it underpredicts variability for advection-dominated cases. On the other hand, the WVM performs better than the CAM when considering all sites and produces better estimates of the cutoff frequency for that case.

\section{SUMMARY AND CONCLUSION}

The cloud advection model was derived to describe the reduction of solar irradiance variability caused by spatial aggregation of irradiance during cloud advection dominated conditions. The model utilizes a transfer function based approach to represent the aggregation. The model demonstrates that the transfer function of a plant can be represented by the Fourier Transform of the plant's spatial distribution. The overall shape of the plant transfer function was observed to be a lowpass filter for all cases, in agreement with other models found in literature. The critical frequencies of the low-pass filter are characterized in part by the Strouhal number, related to plant size and cloud speed.

We applied the model to real data in an attempt to determine whether advection played an important role in representing the variability and the spatial aggregation process. We compared the model with data from the HOPE-Melpitz measurement campaign. A manually identified, $1-\mathrm{h}$ window that exhibited strong cloud advection was

TABLE III. Cutoff frequency, clearness index RMSE metric, and clear-sky index time series correlation coefficient for all windows for which it was possible to compute a cloud speed and direction.

\begin{tabular}{lcccc}
\hline \hline Metric & $\begin{array}{c}\text { CAM } \\
\text { (QC only) }\end{array}$ & $\begin{array}{c}\text { WVM } \\
\text { (QC only) }\end{array}$ & $\begin{array}{c}\text { CAM } \\
\text { (all sites) }\end{array}$ & $\begin{array}{c}\text { WVM } \\
\text { (all sites) }\end{array}$ \\
\hline RMSE $_{f_{c}}$ & 0.0039 & 0.0061 & 0.0024 & 0.0011 \\
RMSE $_{k_{c}}$ & 0.0371 & 0.0604 & 0.0677 & 0.0423 \\
\hline \hline
\end{tabular}

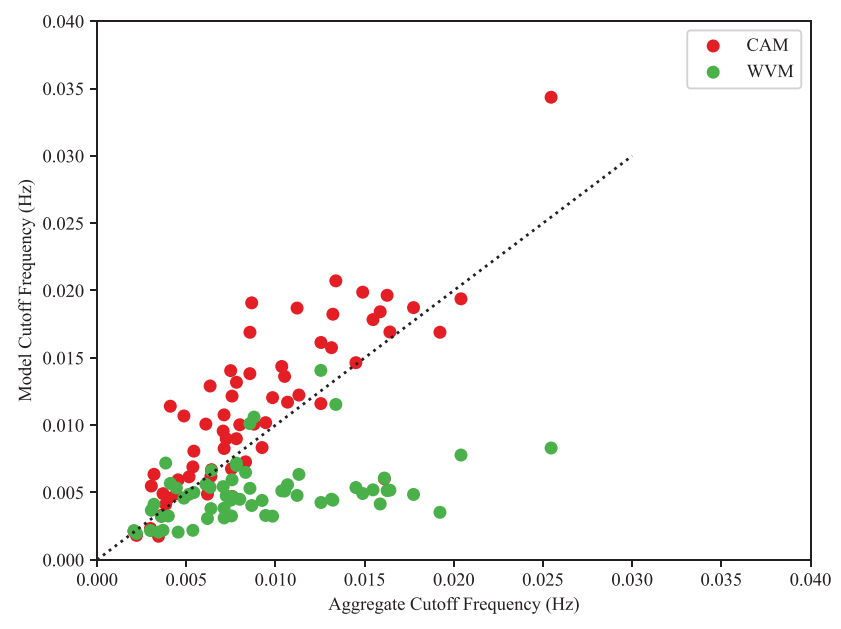

FIG. 13. Comparison of transfer function cutoff frequency calculated at $50 \%$ of base magnitude for all time windows that produced a valid cloud velocity. Transfer function computed for only sites passing quality control.

used as a test case, and a subset of measurement sites were used to simulate a one-dimensional plant. The transfer functions predicted by the cloud advection model agreed quite well with those of the actual data, revealing several important features. The transfer function exhibited a characteristic low-pass filter shape although higher frequency dynamics were observed. The higher frequency dynamics were consistent with spatial interference effects between the various measurement sites, which were unique to the cloud advection model. Varying the subset of measurement sites chosen to represent the plant confirmed that the dynamic characteristics in the transfer function are indeed associated with cloud advection. We confirmed the ability of the transfer function aggregation approach to produce smoothed time series that correspond reasonably well to the validation data. Accounting for delay in the transfer function phase allowed a time series for the whole plant to be produced using any individual reference site.

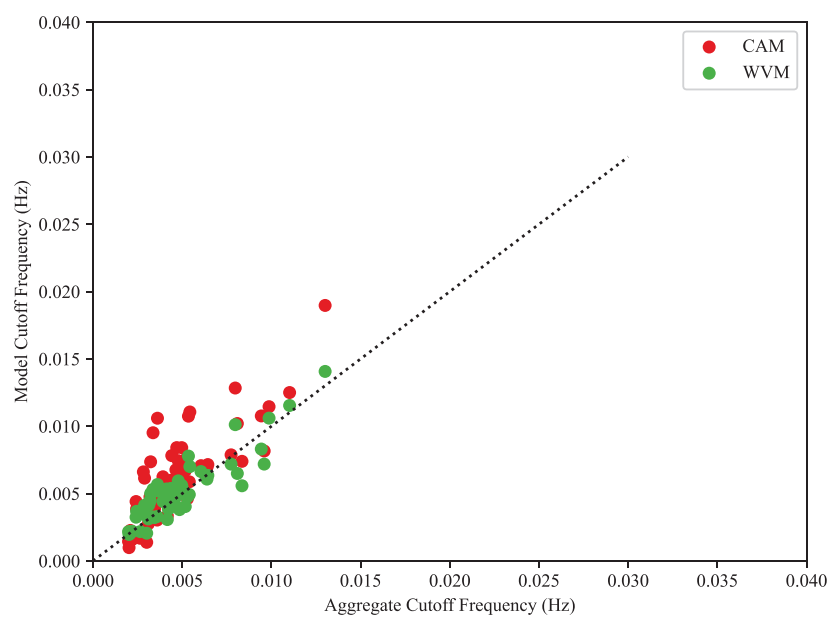

FIG. 14. Comparison of transfer function cutoff frequency calculated at $50 \%$ of base magnitude for all time windows that produced a valid cloud velocity. Transfer function computed for all Melpitz sites. 
The ability of the cloud advection model to predict the value of traditional variability metrics was also demonstrated for the various cases discussed. The cloud advection model provides good prediction of the variability metrics for the one-dimensional, advection dominated conditions. We observed that the RMSE of the clear-sky index time series for the cloud advection model was superior to the WVM and Marcos models for advection-dominated conditions. However, the traditional variance of ramp rate metric was unable to discriminate between the cloud advection model and the Marcos model. Thus, it may be valuable to consider other metrics, including the transfer function, when comparing the quality of model outputs.

While we have utilized short time windows during which solar irradiance can be assumed to behave in a stationary manner in this study, wavelet methods may also be a possible way to account for the actual non-stationary nature of the solar irradiance signals.

In addition to the highly simplified one-dimensional representations, we demonstrated the ability of the model to be applied to twodimensional plants that experience cloud advection. When considering a quality controlled subset of the plant, results were similar to those for the simplified one-dimensional plants. In general, the twodimensional plants tend to dampen the impact of high frequency dynamics in the transfer function. This is a result of the more tightly packed plant distributions that arise from projecting a 2-D plant onto a 1-D distribution. This effect was even more evident when the transfer function was computed using all the available sites in the validation dataset, rather than limiting to those that passed quality control. However, the overall behaviors predicted by the Strouhal number (lower cutoff frequency for larger plants and slower wind speeds) were generally supported. Quantitative metrics show that the cloud advection model has more accurate results than the wavelet variability model when considering sites with a strong cloud motion induced correlation, but that the cloud advection model is less accurate than the WVM when incorporating a broader swath of sites, for which the correlation to the reference site declines.

The cloud advection model's performance was observed to decrease with increasing frequency, corresponding to a loss of transfer function coherence. This may be due to the presence of noise or a loss of correlation in the advection across the plant at these high frequencies. Transfer functions, which allow investigation of the coherence (frequency dependent correlation), may be a useful framework to investigate this effect more deeply.

The key result of this paper can be summarized as follows: when considering aggregation by plants that are aligned with the cloudmotion direction, advection-related effects are present in short-term irradiance time series that are not well represented by traditional models of spatial aggregation. The cloud advection model developed in this paper provides better representation of the aggregate time series in these cases. However, outside of these conditions, the foundational assumptions of the cloud advection model somewhat limit its generalizability. The cloud advection model is based upon an assumption of frozen, one-dimensional cloud advection at a fixed speed. Consequently, its accuracy decreased when considering longer time periods (e.g., for which the cloud speed cannot be assumed to be constant) or when considering plants that were larger in the cross-wind direction. This occurred in part due to cross-wind decorrelation of the time series (i.e., the clouds are not truly 1-D). Traditional decorrelation-based models, such as the WVM, showed better performance than the cloud advection model when dealing with these cross-wind distributions where the uniform, frozen clouds assumption is less likely to hold. In a sense, considering plants with a cross-wind dimension and considering longer-term time windows essentially averages out the advection effects that were observed in this study. That said, we have demonstrated that in order to model the aggregation of irradiance at a detailed level, the advection of clouds cannot be neglected, and models that focus solely on the long term decorrelation between sites are unlikely to yield good short term results. Hoff and Perez ${ }^{3}$ describe a continuum between fully correlated, advection dominated conditions and fully decorrelated, independent measurements. The success of the cloud advection model in representing the high detail aggregation of irradiance suggests that further efforts are warranted that seek to bridge the gap between these conditions. The model described in this study shows that advection dominates the reduction of variability when considering short-term detailed plants. Thus, there is a need for continued research to develop models that are able to represent both the high detailed advective phenomena, while still describing the decorrelated long-term and cross-wind variability of large plants.

\section{ACKNOWLEDGMENTS}

J.R. acknowledges financial support by Penn State Engineering Technology and Commonwealth Engineering and Penn State Hazleton, and appreciates the support of the Carl von Ossietzky University of Oldenburg and the DLR Institute for Networked Energy Systems, who hosted his visiting scientist appointment. Part of this work was supported by the Federal Ministry of Economic Affairs and Energy on the basis of a decision by the German Bundestag (project HyForPV, Grant No. 0350032B).

\section{DATA AVAILABILITY}

The data that support the findings of this study and the demonstration code to run the cloud advection model are openly available in GitHub at https://github.com/jranalli/cloud-advection-model.

\section{REFERENCES}

${ }^{7}$ G. M. Lohmann, "Irradiance variability quantification and small-scale averaging in space and time: A short review," Atmosphere 9, 264 (2018).

2Wang et al., "Worst expected ramp rates from cloud speed measurements," in 46th IEEE PVSC (New York City, New York, 2019). https://ieeexplore.ieee.org/ document $/ 8981213$.

${ }^{3}$ T. E. Hoff and R. Perez, "Quantifying PV power output variability," Sol. Energy 84, 1782-1793 (2010).

${ }^{4}$ R. Perez, M. David, T. E. Hoff, M. Jamaly, S. Kivalov, J. Kleissl, P. Lauret, and M. Perez, "Spatial and temporal variability of solar energy," Found. Trends Renewable Energy 1, 1-44 (2016).

${ }^{5}$ G. M. Lohmann and A. H. Monahan, "Effects of temporal averaging on shortterm irradiance variability under mixed sky conditions," Atmos. Meas. Tech. 11, 3131-3144 (2018).

${ }^{6}$ J. M. Bright, Ramp Rate Analysis for Forecasting Operations in Singapore (IEEE, 2020), p. 7.

${ }^{7}$ K. Bradford, R. Walker, D. Moon, and M. Ibanez, A Regression Model to Correct for Intra-Hourly Irradiance Variability Bias in Solar Energy Models (IEEE, 2020), p. 4.

${ }^{8}$ R. Kharait, S. Raju, A. Parikh, M. A. Mikofski, and J. Newmiller, Energy Yield and Clipping Loss Corrections for Hourly Inputs in Climates with Solar Variability (IEEE, 2020), p. 5. 
${ }^{9}$ G. M. Lohmann, A. H. Monahan, and D. Heinemann, "Local short-term variability in solar irradiance," Atmos. Chem. Phys. 16, 6365-6379 (2016).

${ }^{10}$ M. Schroedter-Homscheidt, M. Kosmale, S. Jung, and J. Kleissl, "Classifying ground-measured 1 minute temporal variability within hourly intervals for direct normal irradiances," Meteorol. Z. 27, 161-179 (2018).

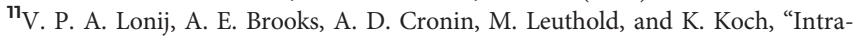
hour forecasts of solar power production using measurements from a network of irradiance sensors," Sol. Energy 97, 58-66 (2013).

${ }^{12}$ L. M. Hinkelman, "Differences between along-wind and cross-wind solar irradiance variability on small spatial scales," Sol. Energy 88, 192-203 (2013).

${ }^{13}$ E. Arias-Castro, J. Kleissl, and M. Lave, "A Poisson model for anisotropic solar ramp rate correlations,” Sol. Energy 101, 192-202 (2014).

${ }^{14}$ G. M. Lohmann, A. Hammer, A. H. Monahan, T. Schmidt, and D. Heinemann, "Simulating clear-sky index increment correlations under mixed sky conditions using a fractal cloud model," Sol. Energy 150, 255-264 (2017).

${ }^{15} \mathrm{H}$. A. Al-Hilfi, F. Shahnia, and A. Abu-Siada, "Comparison of different correlational techniques in estimating the total generated power of neighboring photovoltaic systems," in 2016 Australasian Universities Power Engineering Conference (AUPEC) (IEEE, 2016), pp. 1-6.

${ }^{16} \mathrm{~K}$. Klima and J. Apt, "Geographic smoothing of solar PV: Results from Gujarat,” Environ. Res. Lett. 10, 104001 (2015).

${ }^{17}$ M. Lipperheide, J. L. Bosch, and J. Kleissl, "Embedded nowcasting method using cloud speed persistence for a photovoltaic power plant," Sol. Energy 112, 232-238 (2015).

${ }^{18} \mathrm{~K}$. Shinozaki, N. Yamakawa, T. Sasaki, and T. Inoue, "Areal solar irradiance estimated by sparsely distributed observations of solar radiation," IEEE Trans. Power Syst. 31, 35-42 (2016).

${ }^{19}$ D. Yang, C. Gu, Z. Dong, P. Jirutitijaroen, N. Chen, and W. M. Walsh, "Solar irradiance forecasting using spatial-temporal covariance structures and timeforward kriging," Renewable Energy 60, 235-245 (2013).

${ }^{20} \mathrm{M}$. Jamaly and J. Kleissl, "Spatiotemporal interpolation and forecast of irradiance data using Kriging," Sol. Energy 158, 407-423 (2017).

${ }^{21}$ S. K. Ziyabari, L. Du, and S. Biswas, A Spatio-Temporal Hybrid Deep Learning Architecture for Short-Term Solar Irradiance Forecasting (IEEE, 2020), p. 6.

${ }^{22}$ A. Frimane, T. Soubdhan, J. M. Bright, and M. Aggour, "Nonparametric Bayesian-based recognition of solar irradiance conditions: Application to the generation of high temporal resolution synthetic solar irradiance data," Sol. Energy 182, 462-479 (2019).

${ }^{23}$ A. Frimane, J. M. Bright, D. Yang, B. Ouhammou, and M. Aggour, "Dirichlet downscaling model for synthetic solar irradiance time series," J. Renewable Sustainable Energy 12, 063702 (2020).

${ }^{24} \mathrm{~J}$. Munkhammar, J. Widén, and L. M. Hinkelman, "A copula method for simulating correlated instantaneous solar irradiance in spatial networks," Sol. Energy 143, 10-21 (2017).

${ }^{25} \mathrm{H}$. G. Beyer, "Handling of small scale structures of the irradiance field for solar energy system analysis-A review," Energy Procedia 97, 141-148 (2016).

${ }^{26}$ J. M. Bright, O. Babacan, J. Kleissl, P. G. Taylor, and R. Crook, "A synthetic, spatially decorrelating solar irradiance generator and application to a LV grid model with high PV penetration," Sol. Energy 147, 83-98 (2017).

${ }^{27}$ M. Lave, J. Kleissl, and J. S. Stein, "A wavelet-based variability model (WVM) for solar PV power plants," IEEE Trans. Sustainable Energy 4, 501-509 (2013).
${ }^{28}$ J. Widén, "A model of spatially integrated solar irradiance variability based on logarithmic station-pair correlations," Sol. Energy 122, 1409-1424 (2015).

${ }^{29}$ B. Elsinga, "Chasing the clouds: Irradiance variability and forecasting for photovoltaics," Ph.D. dissertation (Utrecht University, 2017).

${ }^{30} \mathrm{H}$. A. Al-Hilfi, F. Shahnia, and A. Abu-Siada, "Enhancing the estimation of the overall produced power by several adjacent photovoltaic systems using existing correlational factors," in 2018 IEEE 27th International Symposium on Industrial Electronics (ISIE) (IEEE, 2018), pp. 43-47.

${ }^{31}$ A. R. Dyreson, E. R. Morgan, S. H. Monger, and T. L. Acker, "Modeling solar irradiance smoothing for large $\mathrm{PV}$ power plants using a 45-sensor network and the wavelet variability model," Sol. Energy 110, 482-495 (2014).

${ }^{32} \mathrm{~W}$. Holmgren, C. Hansen, and M. Mikofski, "pvlib python: A python package for modeling solar energy systems," J. Open Source Software 3, 884 (2018).

${ }^{33} \mathrm{M}$. Lave and J. Kleissl, "Cloud speed impact on solar variability scalingApplication to the wavelet variability model," Sol. Energy 91, 11-21 (2013).

${ }^{34} \mathrm{~J}$. Marcos, L. Marroyo, E. Lorenzo, D. Alvira, and E. Izco, "From irradiance to output power fluctuations: The PV plant as a low pass filter," Prog. Photovoltaics: Res. Appl. 19, 505-510 (2011).

${ }^{35}$ J. Marcos, I. d 1 Parra, M. Garcia, and L. Marroyo, "Simulating the variability of dispersed large PV plants," Prog. Photovoltaics: Res. Appl. 24, 680-691 (2016).

${ }^{36} \mathrm{~J}$. Ranalli, E. E. Peerlings, and T. Schmidt, Cloud Advection and Spatial Variability of Solar Irradiance (IEEE, 2020), p. 8.

${ }^{37}$ J. A. Ranalli and D. Ferguson, "Convective wave motion during combustion instability in a low-swirl burner flame," in Western States Section of the Combustion Institute Spring Technical Meeting (Western States Section/ Combustion Institute, 2010), pp. 11-19.

${ }^{38}$ A. Macke, P. Seifert, H. Baars, C. Barthlott, C. Beekmans, A. Behrendt, B. Bohn, M. Brueck, J. Bühl, S. Crewell, T. Damian, H. Deneke, S. Düsing, A. Foth, P. D. Girolamo, E. Hammann, R. Heinze, A. Hirsikko, J. Kalisch, N. Kalthoff, S. Kinne, M. Kohler, U. Löhnert, B. L. Madhavan, V. Maurer, S. K. Muppa, J. Schween, I. Serikov, H. Siebert, C. Simmer, F. Späth, S. Steinke, K. Träumner, S. Trömel, B. Wehner, A. Wieser, V. Wulfmeyer, and X. Xie, "The $\mathrm{HD}(\mathrm{CP})^{2}$ observational prototype experiment (HOPE) - An overview," Atmos. Chem. Phys. 17, 4887-4914 (2017).

${ }^{39}$ B. L. Madhavan, J. Kalisch, and A. Macke, "Shortwave surface radiation network for observing small-scale cloud inhomogeneity fields," Atmos. Meas. Tech. 9, 1153-1166 (2016).

${ }^{40} \mathrm{~T}$. Inoue, T. Sasaki, and T. Washio, "Spatio-temporal kriging of solar radiation incorporating direction and speed of cloud movement," in 26th Annual Conference of the Japanese Society for Artificial Intelligence (The Japanese Society for Artificial Intelligence, 2012), p. 1K2IOS1b3.

${ }^{41} \mathrm{M}$. Jamaly and J. Kleissl, "Robust cloud motion estimation by spatio-temporal correlation analysis of irradiance data," Sol. Energy 159, 306-317 (2018).

${ }^{42}$ M. J. Espinosa-Gavira, A. Agüera-Pérez, J.-C. Palomares-Salas, J.-J. Gonzálezde-la Rosa, J.-M. Sierra-Fernández, and O. Florencias-Oliveros, "Cloud motion estimation from small-scale irradiance sensor networks: General analysis and proposal of a new method," Sol. Energy 202, 276-293 (2020).

${ }^{43}$ J. Ranalli (2021). "Cloud advection model," GitHub. https://github.com/jranalli/cloud-advection-model 